David A. Himmelgreen

Department of Anthropology, University of South Florida, Tampa, U.S.A.

dhimmelg@usf.edu

\section{Using a life course approach and a bio-cultural perspective to understand the food insecurity and obesity paradox}

Using cross-sectional data from the $2006 \mathrm{Bra}$ zilian Demographic and Health Survey (DHS), Schlüssel et al. examine the association between household food insecurity (HFI) and excess body weight/obesity among adult women, female adolescents, and children under five. Their results suggest that the nature of the relationship is a function of the life course stage. Historically, HFI has been associated with undernutrition in children and adults (females in particular), especially in poorer countries where adequate nutrition, sanitation, and healthcare are in short supply. More recently however, the paradoxical situation of HFI and overweight and obesity has been demonstrated in high-income, middle-income, and in ascending- or transitional-income countries. In addition to the life course stage, what is particularly interesting about this paradox is that the strength of the relationship between food security status and excess body weight is also influenced by environmental conditions and that there is a synergistic relationship between human biology and culture and behaviors.

In this paper, while the positive association between HFI and child obesity is not statistically significant, food-insecure adolescent females are about two times more likely to have excess weight when compared to their food-secure counterparts. The likelihood of excess weight is nearly 1.5 times higher among food-insecure adult females than among food-secure women. On one hand, adult females with moderate food insecurity are at a $49 \%$ higher risk of being obese than their food-secure counterparts. On the other hand, the risk of excess weight among adolescent females is significantly higher for those with severe HFI (rather than moderate HFI) when compared to their food-secure counterparts.

The authors suggest that the differences in excess weight gain/obesity by severity of food insecurity between adult women and adolescent females reflect that the latter are resistant to fat accumulation because of the physiological changes associated with puberty (e.g., adolescent growth spurt which requires additional energy for increases in skeletal dimensions and developmental changes associated with reproductive maturation). Additionally, the authors point to cultural practices and behavior related to body image and ideal body types as a possible mechanism to explain the difference in 
risk for excess body weight/obesity by the food insecurity severity. Only when HFI is severe for adolescent females does the risk of excess body weight increase substantially because household economics are more constrained and families increasingly purchase high-fat and energy-dense foods. These results strongly suggest that a biocultural perspective is useful in understanding the relationship between HFI and excess body weight/obesity.

After adjusting for several possible confounders, the results for the adult women and adolescent females remained statistically significant. The findings compare reasonably well to studies of HFI and excess weight in higher income countries such as the U.S., suggesting that Brazil is well into the later stages of the nutrition transition as compared to other middle-income Latin American countries.

One of the strengths of this study is its focus on life course stages. While the physiological, genetic, and epigenetic mechanisms are still not well understood, there is mounting evidence showing that early human development during gestation, infancy, childhood, and adolescence are associated with health and well-being in later life. One theoretical framework that may be useful for understanding the way in which food insecurity during different life course stages and in different environmental circumstances (prenutrition transition, nutrition transition, and post-nutrition transition) might increase risk to excess weight gain/obesity is life history theory (LHT). LHT posits that in environments where there is limited energy availability and/or low quality nutrition, trade-offs take place in which growth, development, and immunity are compromised at the expense of reproduction and reproductive fitness. In addition to LHT, the Fetal Origins Hypothesis (FOH) sets out to test whether nutritional and other stresses during gestation are responsible for chronic, degenerative conditions (e.g., heart disease and type 2 diabetes) in adult health. Although there is debate regarding the $\mathrm{FOH}$, there is growing observational evidence that fetal shocks do have the potential to adversely affect health and potentiality later on in life (e.g., test scores, educational attainment, and income) 1,2 .

Together with LHT and the FOH is the idea that humans are complex social beings who attempt to adjust to stressful environmental conditions by employing cultural and behavioral coping strategies that buffer against, for example, nutritional stress. Coping strategies include households shifting away from nutritious foods towards high-fat and energy-dense foods, adult household members cutting back on meals or portion sizes, and increased reliance on familial and community social networks for food assistance. Therefore, a bio-cultural perspective in concert with theories such as LHT and the FOH are likely to bear fruit in terms of the paradox of HFI and excess weight/obesity.

Although this paper uses a sophisticated sampling design, one of the study's main limitations is that the data are cross-sectional. Longitudinal data would allow for testing the hypothesis that HFI increases weight gain over time. Furthermore, since a life course approach is employed, multiple data points through time would permit an analysis where biological and cultural and behavioral variables could be used to predict the risk for excess weight/obesity gain in both foodsecure and food-insecure households. Of course, such data sets are difficult to come by; nonetheless, longitudinal studies need to be conducted on this topic, especially in light of the accumulating evidence about early human development outcomes and later health and well-being. Aside from this limitation, my only other small quibble is with one the confounders used in the multivariate analysis. As an anthropologist, I find the categorization of mother's skin color problematic. While skin color is a social construction with implications regarding racism and access to resources, the five skin colors categories used in this paper are very fluid and without clear demarcation. Moreover, it is not clear to me what "indigenous" or "yellow" skin color means. Unfortunately, this harkens back to outdated racial types with little or no meaning, particularly when it comes to biological variation.

In conclusion, Schlüssel et al. results provide additional support for the nutrition transition in Brazil, the food insecurity and excess body weight/obesity paradox there, and the differential effects of food insecurity on fat accumulation by life course stage. More longitudinal research on this topic is needed and the incorporation of a bio-cultural perspective along with theoretical frameworks such as LHT and the FOH might go a long way in increasing our understanding of the relationship between early human development and health and well-being in later life.

1. Almond D, Currie J. Killing me softly: the fetal origins hypothesis. J Econ Perspect 2011; 25:152-72.

2. Bateson P, Barker D, Clutton-Brock T, Deb D, D'Udine B, Foley RA, et al. Developmental plasticity and human health. Nature 2004; 430:419-21. 